\title{
DIAMOND SHAPED OPTICAL FIBER DELIVERY SYSTEM
}

\author{
Haim Mnitentag, B.Sc, ${ }^{1}$ Prof.Alexander Gersten, Ph.D ${ }^{2}$ \\ and Prof. Shlomo Walfisch, MD ${ }^{1}$
}

1: Colorectal Unit and Laser Center, Soroka Medical Center 2: Physics Department, Ben-Gurion University of the Negev, Beer-Sheva, Israel

\begin{abstract}
Background: Optical fibers are generally used with a flat cut end. The adaptation of a coaxial exit of a refrigerated gas near the distal tip of the optical fiber cools the tissue where the laser beam is applied and reduces the injury of thermal effects on biological tissue.

Materials and Methods: We have changed the flat end geometry of the fiber's end to a pyramidal end shape (named: Diamond tip). In this project we have evaluated the new shape of the fiber's end using laboratory tests and in real conditions. We have examined the emission pattern in the air and in water as well as its performance in surgery.

Results: With the new shape better results were obtained: higher power density, more utilizations of the same fiber (after sterilization) resulting in economical savings. The diamond tip is characterized by greater mechanical resistance then conical tip delivery systems. The greater resistance of the Diamond tip prevents optical fiber breakage during surgery. The delivered system can be reshaped by a trained hospital physician and reused. Our experience shows that delivery systems intended by the producer to one use only can be converted, by reshaping their tips, to highly performing delivery systems, which can be reused many times. In addition less power was needed, with less tissue penetration. The thermal effect was minimized and higher mechanical resistance was achieved, which allowed reusing the fiber more than six times. The special structure of the faces of the optical fiber diamond tip eased its sliding.

Conclusion: The Diamond tip is superior to other fiber end tips by having minimal thermal effects, higher mechanical resistance and by being reused many times.
\end{abstract}

Key Words: Optical fiber, pyramidal end shape, diamond tip, reduced tissue penetration, higher mechanical resistance, reduced thermal effects

\section{INTRODUCTION:}

The use of Nd:YAG and diode laser in surgery, led to the development of fiber optics with different shapes of distal tips. Each tip shape allows specific function, like: excision, vaporization and coagulation of tissue. The use of fiber optics in contact with biological tissue enhances the range of capability of the laser system. It provides significantly increased power density, and allows precise surgery. In the Colorectal Unit we used

Addressee for Correspondence:

Shlomo Walfisch, MD

Colorectal Unit and Laser Center

Soroka University Medical Center

E-mail: walfisch@bgu.ac.il optical fiber with a diamond shaped tip (DIAMOND) with diameter of 600, 800 and 1000 microns. The DIAMOND is characterized by higher mechanical resistance that prevents its breakage during surgery. This delivery system is characterized by greater mechanical resistance then that of other delivery systems. This characteristic prevents optical fiber breakage during surgery and allows its reuse more than six times. While in contact with tissue the DIAMOND provides less tissue penetration, increases the power density and allows precise surgery. The special structure of the faces of the optical fiber diamond tip eases its sliding. When in contact with tissue, this optical fiber is even

Manuscript received: October 13th, 2010

Accepted for publication: January 5th, 2011 
more effective. The adaptation of a coaxial exit of refrigerated gas near the distal tip of the optical fiber cools the tissue where the laser beam is applied and reduces the injury of thermal effects on biological tissue. With this delivery system the thermal effect is minimized, and the cutting quality of the laser is upgraded.

\section{BACKGROUND}

The use of laser and optical fiber to cut and coagulate biological tissues, such as in cases of prolapsed hemorrhoids, complicated peri-anal fistula and condilomata acuminata, gained significant importance in the medical area.

The correct medical usage of these optical devices is determined by the knowledge of their technical performance in real conditions, i.e., in the surgery.

Optical fiber delivery system of laser power to irradiate biological tissue has advantages over other delivery methods because:

1. It gives greater maneuvering freedom to the surgeon than the articulated arm with mirrored reflector system.

2. It allows power to be delivered endoscopically through natural body channels with minimal invasive surgery.

The use of the Nd:YAG laser beam through optical fiber $7,8,10)$ being in contact with biological tissues led to development of different shapes of distal tips such as flat, angled, hemispherical and conical ones. $3,4,9)$ Each tip shape results in a characteristic laser tissue interaction. Many different tip shapes are possible. The different radiation pattern depends on the optical fiber end shape: flat, conical, hemispherical or attaching an optical element to the end. Each shape permits the delivery system to excise, to vaporize, or to coagulate the tissue. ${ }^{2,5,6)}$ The optical fiber delivery system with flat tip profile does not concentrate the laser energy after its delivery, thus it is used to vaporize or to ablate the tissues. It can also be used near the tissue in surgeries performing coagulation. The angled end tip 9) has the same function as the flat tip, but spreads the laser radiation along wider solid angles. The hemispherical end tip has a short focus distance after which the laser beam spreads along a wider solid angle. It provides cutting. The conical end tip offers high concentration of energy, provides excellent cutting as well as coagulation of the tissue under its surface, but it has the disadvantage of breaking and burning easily.

In spite of the assorted availability of delivery systems, the delivery system in current use burns and breaks easily, therefore the laser surgeons are looking for new development with better performance. ${ }^{11)}$ The surgeon may finish the surgery with a damaged delivery system or else he must replace it with a new one - increasing thus the inconvenience and the cost of the surgery.

In order to solve this problem, we have performed surgery with many delivery system prototypes and we have built a new type of delivery system that satisfies the surgeon's requirements. We call it the Diamond Shaped Optical Fiber Delivery System or shortly the DIAMOND. The diamond shaped tip is depicted in Fig. 1.

The performance of the conical tip can be described with the aid of Fig. 2 and an analysis of the internal reflections.
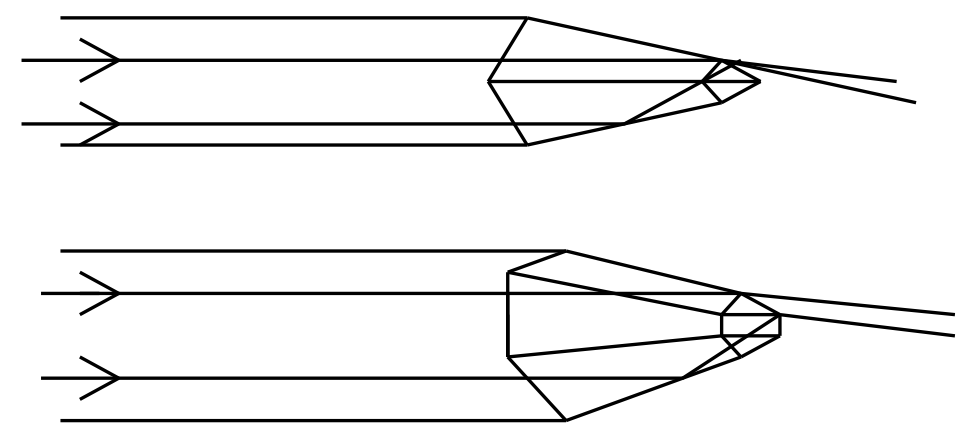

Figure. 1: Diamond Shape Optical Fiber Delivery System 


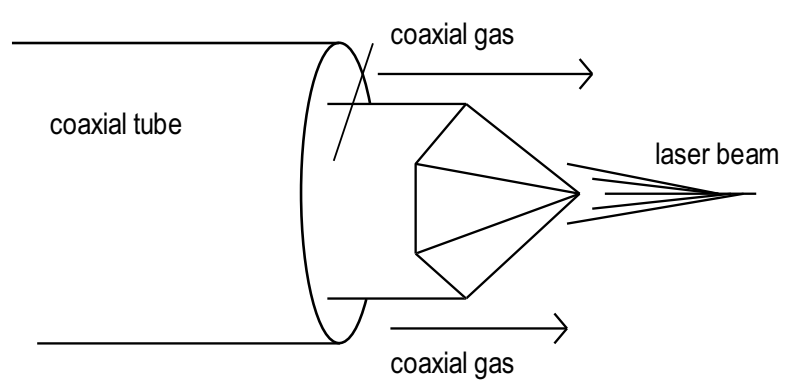

Figure. 2: Diamond delivery system with coaxial cooling

The critical angle Bc (Fig. 3) for internal reflection at $\mathrm{Y}$ is determined by $\arcsin \left(\mathrm{N}^{\prime} / \mathrm{N}\right)$, where $\mathrm{N}^{\prime}$ and $\mathrm{N}$ are indices of refraction of the tip and the surrounding medium, respectively. All rays incident at angles bigger then Bc (Fig. 4) will be totally reflected and contained within the tip. However at the next reflection of the ray at point $\mathrm{X}$, the incident angle will have decreased by the cone angle $\mathrm{C}$, possibly allowing the ray to escape. Subsequent reflections and their angles of incidence also decrease by $\mathrm{C}$, producing a large amount of randomly scattered light.

With the DIAMOND we intended to focus all the random light and the laser beam that naturally is going to leave in the forward direction, ahead of the optical fiber.

For the silica fiber $(\mathrm{N}=1.44)$ in air $\left(\mathrm{N}^{\prime}=1\right)$, the critical angle is $\mathrm{Bc}=44$ and in water $(\mathrm{N}=1.33), \mathrm{Bc}=67.5$

When the tip is immersed in water, the critical angle becomes larger and fewer light rays (compared with the tip in air) are incident at angles greater than the critical angle. Therefore, in water it would be expected that a larger portion of the conical tip would emit light. This would lower the thermal load on the tip and spread any emission pattern. Also in this case the DIAMOND will have better performance, because it spreads the laser beam before the light rays approach the critical angle.

In this project we have developed the DIAMOND and evaluated it in laboratory and in real conditions. We examined the emission pattern of the DIAMOND in the air, immersed in the water and its performance in the surgery.

\section{OBJECTIVES}

\section{Building a delivery system that delivers high power density.}

We have built a Diamond with a polishing angle of $60^{\circ}$, in fibers with 600,800 , and $1000 \mu \mathrm{m}$. We applied Nd:YAG laser, $1064 \mathrm{~nm}, 25$ Watts. Theoretically, the power densities were:

$\begin{array}{cll}\text { FIBER } & \text { FLAT END } & \text { DIAMOND } \\ \text { DIAMETER } & \text { FIBER } & \text { FIBER } \\ 600 \mu \mathrm{m} & 88.5 \mathrm{~W} / \mathrm{mm}^{2} & 442.5 \mathrm{~W} / \mathrm{mm}^{2} \\ 800 \mu \mathrm{m} & 49.8 \mathrm{~W} / \mathrm{mm}^{2} & 249.0 \mathrm{~W} / \mathrm{mm}^{2} \\ 1000 \mu \mathrm{m} & 31.8 \mathrm{~W} / \mathrm{mm}^{2} & 159.0 \mathrm{~W} / \mathrm{mm}^{2}\end{array}$

We measured the laser power with power meter Laserstar, Ophir, Israel.

The larger the fiber diameter was, the less concentrated was the laser beam into the fiber. So the laser reached the fiber end in a larger cross section. Consequently it spread out in larger solid angles, and the power density was diminishing.

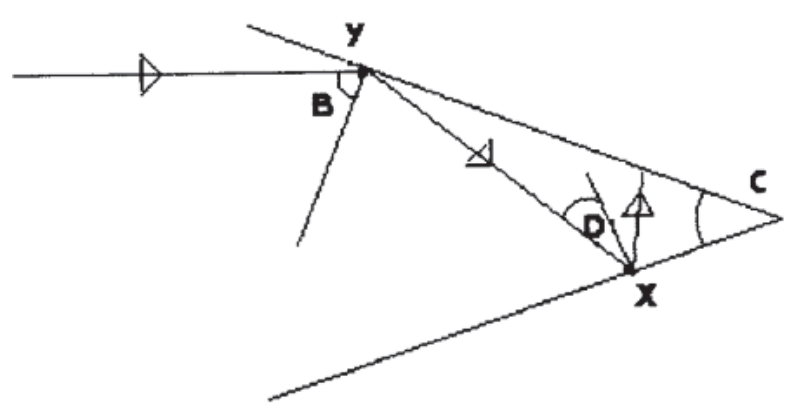

Figure. 4: Geometrical ray tracing of laser beam 
2. Developing a tip with high durability, that can be used numerous times before it becomes necessary to reshape it.

We verified that it is possible to reuse the same DIAMOND in more than five surgeries, after which there was a power loss of up to $25 \%$.

In 119 surgeries, using power of 25 watts and average energy of 2550 joules, we measured the power delivered by the fiber after the proceedings:

\begin{tabular}{l|c|ccc|c}
\hline & $\begin{array}{c}\text { LASER POWER } \\
\text { (W) }\end{array}$ & $\mathbf{6 0 0}$ & $\begin{array}{c}\text { DIAMETER OF FIBER } \\
\mathbf{8 0 0}\end{array}$ & $\mathbf{1 0 0 0}$ & $\begin{array}{c}\text { POWER OUTPUT } \\
\text { (W) }\end{array}$ \\
\hline After the: & & \multicolumn{3}{|c}{ No of FIBERS } \\
1st proceeding: & 25 & 8 & 12 & 5 & 23.7 \\
2nd proceeding: & 15 & 4 & 8 & 3 & 23.2 \\
3rd proceeding: & 8 & 6 & 2 & - & 22.2 \\
4th proceeding: & 5 & 3 & 2 & - & 20.7 \\
5th proceeding: & 4 & 2 & 2 & - & 18.7 \\
\hline
\end{tabular}

\section{To study the relationship between use of this tip and performance.}

With the measurements of power after surgery, we noted that the fibers with $600 \mu \mathrm{m}$ diameter were damaged earlier, and fibers with $1000 \mu \mathrm{m}$ were more lasting, but their lack of flexibility made their handling to be difficult. We observed that fibers with $800 \mu \mathrm{m}$ were at same time flexible and relatively lasting.

We have concluded that the fibers with $600 \mu \mathrm{m}$ were damaged earlier due to their highest power density $\left(442.5 \mathrm{w} / \mathrm{mm}^{2}\right)$ concentrated in their smaller diameter. These fibers in contact with biological tissues produced thermal energy very fast.

The $1000 \mu \mathrm{m}$ fibers are harder to brake. However their larger diameter permitted to produce larger tip end that did not concentrate the power as well as fibers with smaller diameter.

Thus we found an optimum with the $800 \mu \mathrm{m}$ fiber, which gives high power density, mechanical resistance and a flexible system.
4. Comparing the tip thermally and mechanically with others delivery systems.

The Diamond delivery system is characterized by greater mechanical resistance then conical tip delivery systems. Using the conical tip in the same conditions, we observed that it was braking already after the second procedure. The greater resistance of the Diamond prevents optical fiber breakage during surgery.

The DIAMOND delivers almost all laser energy through the points that are in contact with tissue. At these points the tissue is evaporated but afterwards the spread through the tissue is diminished allowing thus a precise and minimally invasive surgery.

After the surgery, the fibers are being cleaned and sterilized for subsequent reuse according to the high performance level which characterizes the new system. This procedure was reused and tested on one fiber more than six times during colorectal surgery with excellent results.

We have adapted a coaxial exit of cooling gas, near the distal tip of the optical fiber, as depicted in Fig. 2. That cooling of the tissue around the laser beam reduced significantly the thermal injury. In this way the characteristics of the DIAMOND were significantly improved. 


\section{MATERIALS AND METHODS}

\section{Laboratory}

In our unit we tapered the end of the optical fiber (with diameters of $600,800,1000$ micron) to a diamond shape using the polishing machine Ultratec, using fluids and pads. We used silica clad - silicon core optical fiber. The fibers were tested with a $3 \mathrm{~mW} \mathrm{HeNe}$ laser beam $(630 \mathrm{~nm})$ in order to observe the spreading of the irradiation.

A continues wave Nd:YAG laser system (model 3000, Laser Industry, Israel) with a wavelength of $1064 \mathrm{~nm}$ was used. During surgeries the laser was applied with a power of 25 Watts in a continuous mode, the mean time of use was 102 seconds and the averaged delivered energy was 2550J. We used a HeNe laser beam (630nm) with $5 \mathrm{mWatts}$ as aiming beam. The power delivered through the optical fiber was within $10 \%$ of the setting power of the display.

The laser power was measured by OPHIR LaserStar Power Meter.

We have produced the DIAMOND delivery system from a bare optical fiber by stripping its $10 \mathrm{~mm}$ clad of silicone and shaping the faces on its distal end with angles of $60^{\circ}$. We used a precision manipulator and a high quality polishing system (Ultratec). The surface of the faces received a high grade of polishing to avoid heating and adherence of biological material during the surgery and to efficiently deliver the laser beam.

\section{Theoretical Analysis}

Indications of the optical performance of optical fiber tips can be obtained from thick Lens Theory, ${ }^{13)}$ Matrix Optics ${ }^{15)}$ and Total Internal Reflection. 16)

\section{RESULTS}

The Colorectal unit has used the DIAMOND in 119 surgeries, ${ }^{1)}$ performing excision of pilonidal sinus and of condiloma, hemorrhoidectomies, fistulectomies and sphincterotomies.

Transmission measurements indicate that the DIAMOND transmits $85 \%$ of $\mathrm{Nd}$ :YAG radiation. Correspondent transmission of spherical tips is 90\%. ${ }^{12}$ )

We have compared the effect of cutting of the DIAMOND with the cutting of a flat, conical and angled shape tips. The results were quite satisfactory. The increased mechanical resistance of the optical fiber can be estimated by the number of surgeries performed with the same fiber. We performed six surgeries with the same fiber with the DIAMOND delivery system, working at a high performance level. Conical types of delivery systems broke after two or three surgeries. Flat types normally were burned after the first surgery. These findings indicate that we have better performance, better mechanical resistance and durability with the DIAMOND in comparison with other delivery systems.

\section{DISCUSSION}

Physicians using laser systems are looking for new developments of delivery systems with better performance, since the systems found in the market burn and break easily. Occasionally, the surgeon finishes the surgery with a delivery system that is damaged or has to switch to a new one. In order to overcome the above disadvantages, we developed several new prototypes of optical fiber delivery systems which were tested in surgical procedures. On the basis of our observations we developed a new prototype of delivery system that we called Diamond Shaped Optical Fiber Delivery System (DIAMOND). It seems that the performance of the DIAMOND fits the requirements of the surgeons.

We think that the greater resistance of the end tip with diamond geometry is due to the mechanical distribution of forces on the tip. Additional support is obtained by the fiber's nylon covering which gives it greater flexibility and breakage prevention along the fiber's length. The surfaces of the diamond faces have received a high grade of polishing in order to avoid heating and adherence of biological material during the surgery and in order to deliver the laser beam with efficiency and ease.

Although the laser radiation transmission of the DIAMOND is smaller than the spherical one, the energy density of the DIAMOND is bigger than the spherical, so its cutting and vaporizing is superior than the spherical.

\section{CONCLUSIONS}

One of the reasons that surgeons do not use lasers is that their purchase and operation are quite expensive. One way of alleviating this problem is by reducing their operational cost. The delivered system can be 
reshaped by a trained hospital physician and reused. Our experience shows, those delivery systems intended by the producer to one use only, can be converted by reshaping their tips, to highly performing delivery systems, which can be reused many times.

The grant received from the Harry Stern \& Helen Tzoref Applied Research Program contributed significantly to the progress of the fiber optics utilities at the Soroka Medical Center. New equipment was purchased which improved the elaboration of the tip of the fiber optics leading to better performances during opera- tions by increasing the life-time and safety use of the fiber optics. Significant savings to the Soroka medical Center were obtained by the reuse of improved fiber optic endings which otherwise were designated to single use only. The estimated savings amount to above $\$ 100,000$. The purchased equipment may serve as a basis for starting a project within the industrial incubators. The project may be headed by Mr. Haim Mnitentag.

Thank you for the financial support: HARRY STERN \& HELEN TZOREF APPLIED RESEARCH PROGRAM

\section{REFERENCES}

1: Walfisch S. et al. Nd:YAG laser For Anorectal Surgery: Initial Experience in Israel. Harefuah, 126:1-4 (1994)

2: Optical Fibers in Medicine, editor: Abraham Katzir. SPIE Milestone Series, Vol. MS11. (1993)

3: Lasers in General Surgery, editor: Stephen N. Joffe, MD., Williams \& Wilkins (1989)

4: Mnitentag H., et al. Laser Surgery in Enclosed Spaces. Lasers in Surgery and Medicine, 5:199-218 (1985)

5: Mnitentag H., et al. Thermoghraphic Study of Laser on Arteries. Lasers in Surgery and Medicine, 7: 307329 (1987)

6: Nolsoe C., et al. Bare Fiber Low Power Interstitial Hyperthermia. Lasers in Medical Science, 7:17(1992)

7: Mnitentag H., et al. Application of Laser Beam through Endoscopy. Gastroentereology, Digestive Endoscopy, 4(1): Jan-Mar(1985)

8: Bigio I.J. et al. Proceedings of Advances in Laser and Light Spectroscopy to Diagnose Cancer and

other Diseases, Progress in Biomedical Optics Vol. 2135, P.26 (1994).

9: Kabalin J.N., Laser Prostatectomy Performed With a Right Angle Firing Neodymium:YAG Laser Fiber at 40 Watts Power Setting. The Journal of Urology, 150: 95-99 (1993)

10: Endoscopic laser Surgery Handbook. Shapshay S.M., Marcel Dekker, Inc. (1987)

11: Medical Lasers. Carruth J.A.S \& McKenzie A.L, Adam Hilger Ltd (1986)

12: Washida H. et al., The Contact Nd:YAG laser System in the Treatment of Bladder Cancer. Lasers Surg. Med. 7, 524 (1987).

13: Fundamental of optics. Jenkins F.A. and White H.E. (McGraw-Hill, New York, 1976)

14: Introduction to Matrix Methods in Optics. Gerrard A. and Burch J.M. (Wiley, New York, 1975)

15: Hand book of Optics (Fiber Optics). Driscol and Vaughn (Siegmund W.P.). McGraw-Hill, New York, 1978. 